\title{
MARKOV RANDOM FIELD IMAGE PRIOR MODELS FOR MAP RECONSTRUCTION OF MAGNETOENCEPHALOGRAM IMAGES
}

\author{
Brian D. Jeffs $\dagger$ and Alan H. Gardiner \\ † Brigham Young University, 459 CB, Provo, UT 84602, email bjeffs@ee.byu.edu \\ $\ddagger$ Lockheed Martin Federal Systems, email: alan.gardiner@lmco.com
}

\begin{abstract}
In this paper the maximum a posteriori (MAP) reconstruction of magnetoencephalograms (MEG) is investigated. The solution is cast as a classical inverse imaging problem, which for MEG is notoriously ill posed and requires strong regularization. Two different Markov random vector field (MRF) models suitable for MEG regularization are developed. The first model uses a potential function which encourages solution sparseness on a rectilinear sample grid. The second model permits simulating an MRF over the non-uniform grid required for hemispherical sampling of the brain. Both methods utilize a new Markov vector field structure where neuron current dipole orientations are explicitly included in the model. MAP reconstructions are presented using simulated and real MEG data.
\end{abstract}

\section{INTRODUCTION}

We will consider maximum a posteriori (MAP) reconstruction of magnetoencephalogram (MEG) data to form neuron electrical activity functional mapping of the human brain. A simple linear relationship between source current dipoles arising from neuron activity and the resulting extracranial magnetic fields is used so that the solution may be cast as a classical inverse imaging problem. A mathematical framework for Markov random field models (MRF) suitable for MEG imaging will be developed.

Neuromagnetic imaging (NMI), the process of creating a source image consistent with the magnetic field observations, was first performed on a single plane with constrained source orientations by Singh et al. [1]. Currently, one of the leading areas of research in NMI is in the Bayesian context, with MRF's used to represent prior knowledge about the source distribution in the form of an image prior probability density function (pdf). In the NMI framework, Phillips et al have developed a composite, dual MRF model using a binary indicator process and independent Gaussian random variables to represent whether a source is on or off and source amplitude respectively [2]. Their model assumes a prior knowledge of source orientation, so a scalar MRF may be used.

In each of the two proposed methods described below, a single consistent vector field MRF source model is intro-

\footnotetext{
${ }^{0}$ The authors express appreciation to Dr. John Mosher and the Albuquerque NM Veterans Administration Hospital for providing data and guidance.
}

duced. In the first method, a Gibbs distribution potential function is chosen to encourage solution sparseness. The second method permits Gaussian MRF modeling on the non-uniformly sampled hemispherical surface used to represent the brain cortex. In both methods, the brain neuron current activity is modeled as an MRF vector field, rather than the conventional scalar field. With the the new vector field approach, dipole orientations are included in the model and are not required to be known a priori.

Neuron electrical activity in the brain will be modeled as a distribution of discrete current dipoles, $\vec{q}_{s}$, each with an unknown magnitude and 3-D orientation. The goal of MEG imaging is to estimate the values of these dipole parameters on a sample grid throughout the tissue of interest. Neuron current dipole activity is observed indirectly using an array of sensitive SQUID magnetometers placed around the skull.

We define the $3 \times 1$ element transfer vector, $\Gamma_{i, s}$, to sat isfy the static Biot-Savart law for single sensor response to an array of active dipoles,

$$
b_{i}=\sum_{s} \Gamma_{i, s} \cdot \vec{q}_{s}, \quad \Gamma_{i, s}=\frac{\mu_{0}}{4 \pi} \frac{\left(\mathbf{r}_{i}-\mathbf{r}_{s}\right) \times \vec{v}(i)}{\left|\mathbf{r}_{i}-\mathbf{r}_{s}\right|^{3}}
$$

where '.' and ' $x$ ' represent vector inner and outer products respectively, $b_{i}$ is the $i^{\text {th }}$ MEG sensor output, $\mathbf{r}_{i}$ is its $3-\mathrm{D}$ position vector, $\vec{v}(i)$ is the unit direction vector normal to the plane containing the sensor's gradiometer coil, $\vec{q}_{s}=\left[q_{s, x}, q_{s, y}, q_{s, z}\right]^{T}$ is the 3-D current dipole vector at the $s^{t h}$ pixel site, and $\mathbf{r}_{s}$ is the position vector for this dipole. The entire array observation may be represented in matrixvector product form.

$$
\begin{aligned}
\mathbf{b} & =\mathbf{G} \mathbf{q}+\eta \\
\mathbf{b} & =\left[b_{1}, \cdots, b_{M}\right]^{T}, \\
\mathbf{G} & =\left[\begin{array}{ccc}
\Gamma_{1,1}^{T} & \cdots & \Gamma_{1, S}^{T} \\
\vdots & & \vdots \\
\Gamma_{M, 1}^{T} & \cdots & \Gamma_{M, S}^{T}
\end{array}\right], \\
\mathbf{q} & =\left[\vec{q}_{1}^{T}, \cdots, \vec{q}_{S}^{T}\right]^{T},
\end{aligned}
$$

where $\eta$ represents sensor error (noise), and $M$ and $S$ are the total number of sensor and pixel sites respectively.

MEG imaging is known to be a notoriously ill posed inverse problem, with a very large ambiguity in the solution space. The proposed algorithms are based on the Bayesian MAP image restoration method because of the 
natural means it provides for regularizing the inverse solution. The MAP estimate of $q$ is given by

$$
\hat{\mathbf{q}}=\arg \max _{\mathbf{q}} f_{q \mid b}(\mathbf{q} \mid \mathbf{b})=\arg \max _{\mathbf{q}} f_{b \mid q}(\mathbf{b} \mid \mathbf{q}) f_{q}(\mathbf{q})
$$

where $f(\cdot)$ represents a probability density function (pdf). The image prior pdf, $f_{q}(\mathbf{q})$, must be chosen to model statistical characteristics of the desired true image, so as to yield a unique solution. We will introduce a two MRF models for $f_{q}(\mathbf{q})$ which significantly improve reconstruction results.

\section{AN MRF MODEL FOR SPARSE MEG REGONSTRUCTION}

For this first algorithm, the image prior pdf, $f_{q}(\mathbf{q})$, is represented by a Gibbs distribution formulation of an edge preserving Markov random vector field model

$$
\begin{aligned}
\log f_{q}(\mathbf{q}) & \propto-\gamma_{1} \sum_{\langle s, t\rangle \in \mathcal{C}} b_{s t} \frac{1}{1+\left(q_{s}-q_{t}\right)^{2}} \\
-\gamma_{1} & \sum_{s \in S} a_{s} \frac{1}{1+q_{s}^{2}}-\gamma_{2} \sum_{\langle s, t\rangle \in \mathcal{C}} \beta_{s t}\left[\left(\theta_{s}-\theta_{t}\right)_{((-\pi, \pi))}\right]^{2}
\end{aligned}
$$

where $q_{s}=\left\|\vec{q}_{s}\right\|, \theta_{s}=\tan ^{-1} \frac{q_{s, y}}{q_{s, x}}$, and subscript ' $((-\pi, \pi))$ ' indicates modulo $2 \pi$ arithametic mapped onto the range $(-\pi, \pi) . \mathcal{C}$ is the set of all pixel cliques in the MRF neighborhood system, and $\mathcal{S}$ is the set of all image sample points. $\gamma_{1}$ and $\gamma_{2}$ are the regularization constants for the magnitude prior and orientation prior respectively. $b_{s t}$ and $\beta_{s t}$ are neighborhood influence parameters that control correlations in magnitude and orientation respectively for neighboring dipoles. Note that for simplicity we have constrained the dipole orientation to lie within the $x-y$ plane, though the extension to $3-\mathrm{D}$ rotation is trivial.

The proposed prior model given in equation (4) incorporates a generalization of the potential function used by $\mathrm{Ge}-$ man et al for single-photon emission tomography [3]. This potential has been shown in scalar fields to produce edgepreserving reconstructions, and we will demonstrate that when used in the MEG vector MRF model it favors the sparse solutions needed for resolving current dipoles. With the dipole field, it is not sufficient to merely control the correlation structure of the pixel magnitudes. Correlated neighboring dipoles should not only have similar magnitudes, but their direction orientation must be similar. The final summation term in equation (4) encourages local homogeneity in dipole orientation by imposing a square law penalty on angular differences.

Assuming i.i.d. Gaussian noise, and substituting equation (4) into (3) yields the restoration solution equation

$$
\begin{aligned}
\hat{\mathbf{q}}= & \arg \min _{\bar{q}}(\mathbf{b}-\mathbf{G} \mathbf{q})^{T}(\mathbf{b}-\mathbf{G q}) \\
& +\gamma_{1}\left(\sum_{(s, t) \in \mathcal{C}} b_{s t} \frac{1}{1+\left(q_{s}-q_{t}\right)^{2}}+\sum_{s \in S} a_{s} \frac{1}{1+q_{s}^{2}}\right) \\
& +\gamma_{2} \sum_{\langle s, t\rangle \in \mathcal{C}} \beta_{s t}\left[\left(\theta_{s}-\theta_{t}\right)_{((-\pi, \pi))}\right]^{2}
\end{aligned}
$$

We have used the Metropolis algorithm for simulated annealing to solve ( 5 .

Figure 1 shows the configuration of simulated MEG sensors on a hemisphere surrounding a $4 \times 4$ grid of current dipole sites. This geometry was used to generate synthetic data to evaluate the effectiveness of the proposed algorithm. A first order uniform neighborhood was used in the MRF model, i.e. for a given pixel site $s, b_{s t}$ and $\beta_{s t}$ were non zero only for indices, $t$, corresponding to the four nearest neighbors (up, down, left, right).

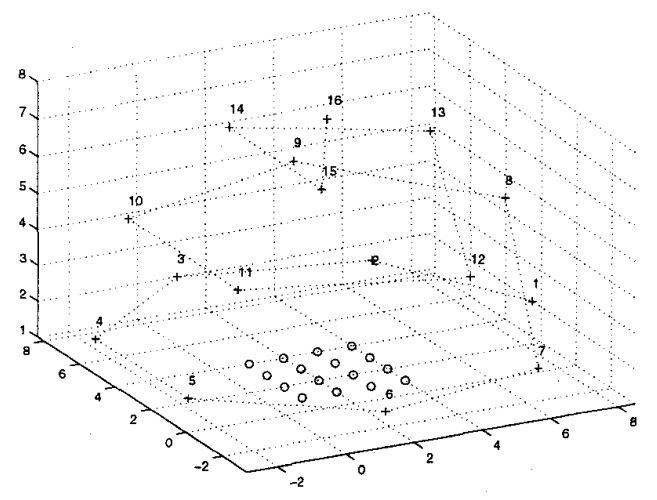

Figure 1. Sensor locations in relation to source locations. Pluses show the sensor array in relation to source locations (circles).

Figure 2 presents a typical example of the restoration cases evaluated for the new algorithm. Figure 2a shows the true dipole locations and orientations used to synthetically compute observed data, b. Figure $2 c$ shows the restoration result using equation (5), while $2 \mathrm{~b}$ presents a minimum norm solution obtained with the ART algorithm for comparison. Clearly the new method beter resolves the two isolated dipoles, and does not suffer from the spurious dipole artifacts that appear in $2 b$. It is noteworthy that both these solutions are equally consistent with the observed data, which illustrates the ill-posed nature of the problem.

\section{NON-UNIFORMLY SAMPLED SCALAR GAUSS-MARKOV RANDOM FIELDS}

Figure 3 shows the actual sensor geometry for the Neuromag model 122 SQUID detector head array that we will use. There are 122 conformal gradiometers, with two mutually orthogonal loop pairs at each of the 61 sites. With this realistic sensor configuration, and forming current dipole estimates on the brain cortex surface, it is not possible to use the rectilinear source grid of the previous section. The dipole sample grid must have non-uniform spacing due to the spherical surface geometry, and therefor a nonuniformly sampled MRF model is called for.

In the absence of detailed brain cortical structure information, and since brain tissue is homogeneous at the scale used for the dipole grid, we are obliged to adopt a homogeneous model for the covariance structure of the current dipole distribution. We will assume that the underlying 
a)

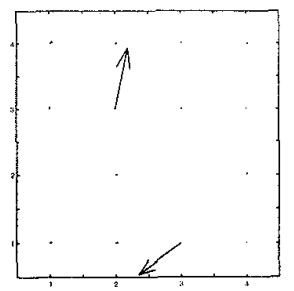

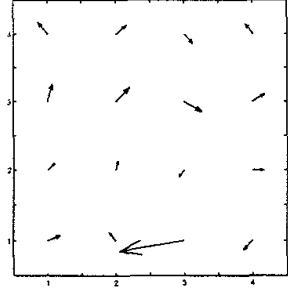

b)

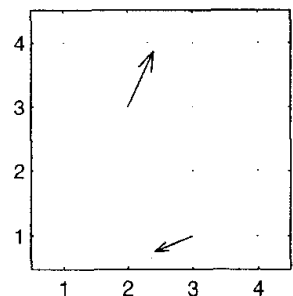

c)

Figure 2. MAP reconstruction of current dipole distribution using a sparse image prior. a) Original current dipole image. b) Minimum norm reconstruction using the ART algorithm. c) MAP reconstrution using prior of equantion (4).

physical current distribution in brain tissue is a stationary, zero mean, continuous space Markov random field [4] with an isotropic covariance structure.

This continuous MRF is sampled at arbitrary points to provide elements of a discrete MRF for our reconstruction model. The correlations between samples are set to match those of the underlying continuous field, but the resulting discrete MRF will in general be neither stationary (with respect to the ordering of sample indices) nor isotropic.

Let $R_{q}(\psi, \tau)$ be the desired covariance function between two continuous space points, $\psi$ and $\tau$, on the dipole hemisphere. By isotropic, we mean that $R_{q}(\psi, \tau)$ is only a function of the Euclidean distance between $\psi$ and $\tau$. Now if $s$ and $t$ are points on the non-uniformly sampled lattice, $\mathcal{S}$, then we require that the covariance between these samples

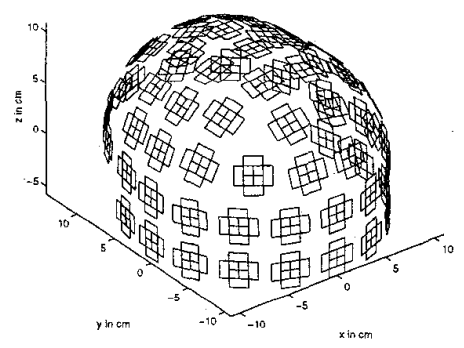

Figure 3. Plot showing gradiometer locations and configurations for the Neuromag 122 system. in the discrete MRF, $\mathbf{q}$, be given by $E\left\{\vec{q}_{s} \cdot \vec{q}_{t}\right\}=R_{q}(\psi=$ $\mathbf{r}_{s}, \tau_{t}=\mathbf{r}_{t}$ ).

The joint pdf of a scalar Gauss-Markov random field, $\mathbf{x}$ can be expressed as $[5,6]$

$$
\log f_{X}(\mathbf{x}) \propto-\left(\sum_{s \in S} a_{s} x_{s}^{2}+\sum_{\{s, t\} \in \mathcal{C}} b_{s t}\left|x_{s}-x_{t}\right|^{2}\right)
$$

Neighborhood influence parameters, $b_{s t}$ and $a_{s}$ in (6) must be chosen to model the desired covariance of the continuous spatial distribution. In uniformly sampled MRF's, this is easily accomplished given a desired covariance matrix, $\mathbf{R}_{x}$, however, no such method is available for non-uniformly sampled fields.

Equation (6) can also be represented as a multivariate jointly normal density with covariance matrix $\mathbf{R}_{x}=\mathbf{B}^{-1}$, where interaction matrix $\mathbf{B}$ has elements, $\left\{B_{s t}\right\}$, which relate to our GMRF influence parameters as follows: $a_{s}=$ $\sum_{t \in S} B_{s t}$ and $b_{s t}=-B_{s t}$ [5]. In a 1-D MRF, an exponential covariance function yields a tri-diagonal $\mathbf{B}$, where most $B_{s t}=0$. Unfortunately, with non-uniform sampling, even the simplest of 2-D continuous covariance functions, $R_{x}(\psi, \tau)$, require nearly all the $B_{s t}$ to be non-zero [7]. An MRF model is only computationally useful if the neighborhood system is small, i.e. if each pixel has only a few neighbors. With most $B_{s t} \neq 0$, every pixel is a neighbor of every other pixel, and the MRF model is useless for efficient reconstruction algorithms. We will present a method for approximating $\mathrm{B}$ for a specified $R_{x}(\psi, \tau)$ such that the neighborhood system remains small, and the model matches the desired correlation structure more closely.

The goal is to find an approximation, $\hat{\mathbf{B}}$, for $\mathbf{B}$ such that: a) most of the $\hat{B}_{s t}=0$, i.e. the neighborhood structure is small, b) $\left\|\hat{\mathrm{B}}^{-1}-\mathbf{R}_{x}\right\|_{F} \leq \varepsilon$, i.e. the approximation error is bounded, c) the element-wise variances of $\mathbf{x}$ match the desired values, and d) $\hat{\mathbf{B}}$ is positive definite, invertable. We have shown that the following approximation meets these criteria $[7]$

$$
\begin{aligned}
& \hat{\mathbf{B}}=\mathbf{D}^{\frac{1}{2}} \mathbf{A} \mathbf{D}^{\frac{1}{2}} \\
& \mathbf{A}=\mathbf{M} \odot \mathbf{R}_{x}^{-1}+\mathbf{E} \\
& \mathbf{D}=\operatorname{diag}\{\mathbf{A}\} \operatorname{diag},\left\{\mathbf{R}_{x}\right\}
\end{aligned}
$$

where $\odot$ indicates the Schur element-by-element matrix product, $\operatorname{diag}\{\}$ is a diagonal matrix formed from the diagonal of the argument. $\mathbf{M}$ is a selection matrix which controls the size of the neighborhood for each pixel, and has elements $m_{s t}$ given by

$$
m_{s t}= \begin{cases}1 & t \in \delta_{s} \\ 0 & \text { otherwise }\end{cases}
$$

where $\delta_{s}$ is the set of all sites which are neighbors of pixel $s$. A simple threshold test is used to form this set. For a given pixel $s, t$ is a neighbor of $s$ if $\left|B_{s t}\right| \geq \tau . \tau$ is manually selected to be just large enough to achieve the desired neighborhood size on average across the sample grid. $\mathbf{E}$ is a diagonal matrix with elements $e_{s s}=\sum_{t \notin \delta_{s}} B_{s t}$. The effect 


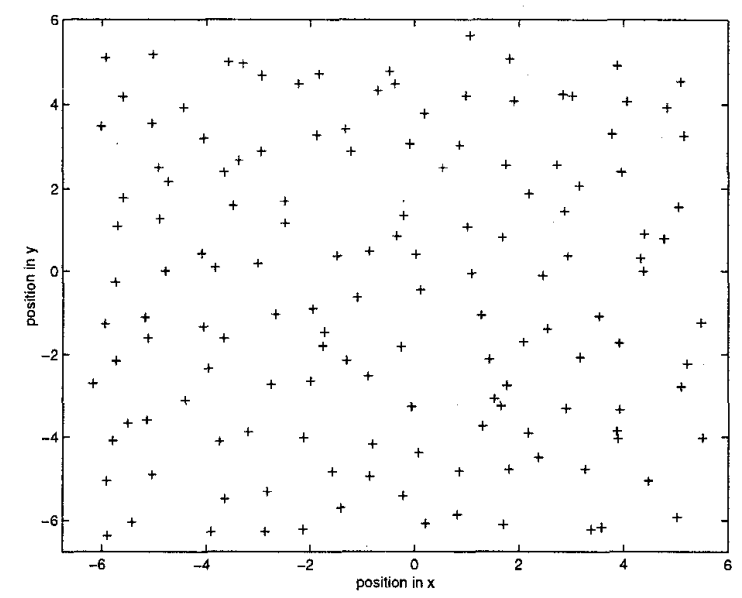

Figure 4. Pseudo-randomly sampled field used in the experiment of Figure 5.

of $\mathbf{E}$ is to insure $\hat{\mathbf{B}}$ will be positive definite and to assist in keeping the pixel marginal variances at the desired level.

It may be noted that forming $\hat{\mathbf{B}}$ requires a one-time computation of the inverse of a potentially very large matrix, $\mathbf{R}_{x}$. In the MEG imaging problem the number of voxels in the image space is relatively small, so this issue is not a problem. Conversely, the space matrix $\hat{\mathbf{B}}$ is preferred to the full $\mathbf{B}$ to reduce neighborhood size and computation load in the iterative algorithms used to solve equation (3).

The effectiveness of the above approximation was demonstrated on a non-uniformly sampled Gauss-Markov random field. A simple 2-D isotropic exponential covariance was used for the underlying continuous field,

$$
R_{x}(\psi, \tau)=\rho^{\||\psi-\tau|_{2}}
$$

The corresponding desired discrete interaction matrix, $\mathbf{B}$, is given by

$$
\begin{aligned}
\left\{\mathbf{B}^{-1}\right\}_{s t} & =\left\{\mathbf{R}_{x}\right\}_{s t}=R_{x}\left(\psi=\mathbf{r}_{s}, \tau=\mathbf{r}_{\mathbf{t}}\right) \\
& =\rho^{\left\|\mathbf{r}_{s}-\mathbf{r}_{\mathbf{t}}\right\|_{2}}
\end{aligned}
$$

Figure 4 shows positions of 144 random samples sites which overlay the exponential correlation continuous MRF. Using these sample locations, the approximation of equation (7) was applied to compute the GMRF neighborhood weights, with $\rho=0.5$, and $\tau$ set to give approximately four neighbors for each pixel site. Chen's algorithm [8] was used to generate 2000 realizations of the GMRF random field so that sample covariances could be computed between pixel sites and compared with the desired values given by $\mathbf{R}_{x}$. Figure 5 compares covariance values (relative to an interior pixel at $s=30$ ) for a synthesized non-uniform GMRF with the corresponding desired values. Note that the synthesized data has sample cross covariance values that track the ideal desired values remarkably closely, with a model neighborhood the same size as a uniformly sampled first order neighborhood.

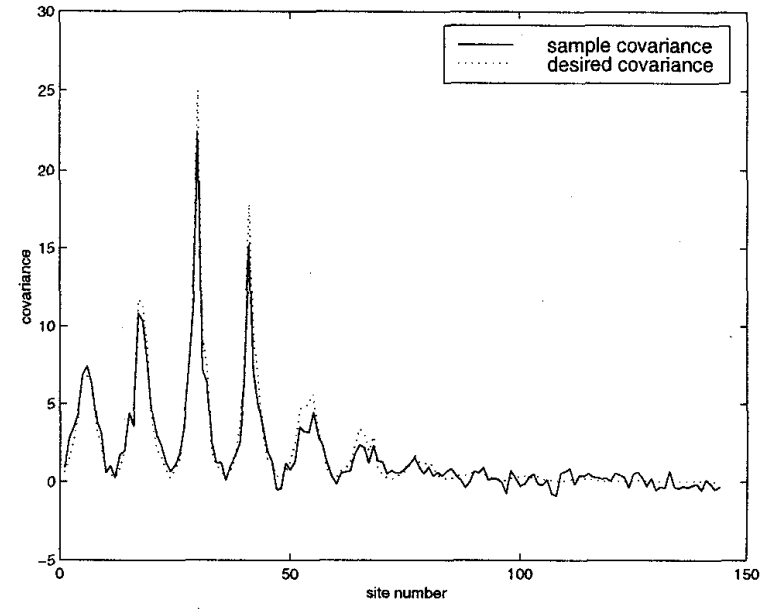

Figure 5. Sample covariance vs. desired covariance for an interior pixel in the field with high sampling randomness.

\subsection{Extension to Vector field Models for MEG Reconstruction}

The vector GMRF model is an obvious extension of scalar equation (6). Note that the second term in this equation is a penalty (in the exponent of $f_{x}(\mathbf{x})$ ) for dissimilar intensity values between pixels $x_{s}$ and $x_{t}$. For a vector field, we wish to penalize (in a square law sense) differences in the vectors at sites $s$ and $t$ to encourage neighboring dipoles to have similar magnitudes and orientations. We propose the following model,

$$
\log f_{q}(\mathbf{q}) \propto-\sum_{s, t \in \mathcal{C}} b_{s t}\left(\vec{q}_{s}-\vec{q}_{t}\right)^{T}\left(\vec{q}_{s}-\vec{q}_{t}\right)-\sum_{s \in S} a_{s} \vec{q}_{s}^{T} \vec{q}_{s} .
$$

Assuming white Gaussian noise, the vector GMRF model of equation (11) for $f_{q}(\mathbf{q})$ leads to the MAP reconstruction solution

$$
\begin{aligned}
\hat{\mathbf{q}}= & \arg \min _{\vec{q}}(\mathbf{b}-\mathbf{G q})^{T}(\mathbf{b}-\mathbf{G q}) \\
& +\lambda \sum_{s, t \in \mathcal{C}} b_{s t}\left(\vec{q}_{s}-\vec{q}_{t}\right)^{T}\left(\vec{q}_{s}-\vec{q}_{t}\right)+\lambda \sum_{s \in \mathcal{S}} a_{s} \vec{q}_{s}^{T} \vec{q}_{s}
\end{aligned}
$$

where $\lambda$ is the regularization weight which controls the relative influence on the solution of the image prior $f_{q}(\mathbf{q})$. Equation (12) can be solved using a simple steepest descent technique.

\subsection{Results with Clinical Data}

Figure 7 shows results of image reconstruction using equation (12) on clinical data collected using the Neuromag 122 array. This data represents the somatosensory evoked response from electric stimulation of the right ring finger. Data is averaged over 109 trials to increase SNR, which even after averaging is less than $10 \mathrm{~dB}$. Figure 6 presents an aggregate plot of the averaged data from all 122 sensors overlaid on a single axis. The sampling interval is $0.8 \mathrm{~ms}$, and the spikes at about sample 125 are induced noise from the shock stimulus. The evoked response occurs at about sample 180 . Note the poor SNR even in this averaged data, 
which makes the results of Figure $7(a, b)$ the more impressive.

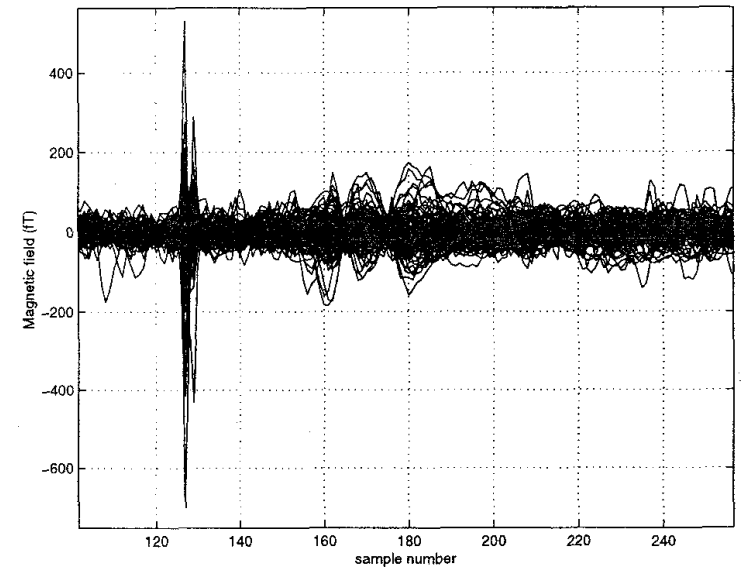

Figure 6. Plot of MEG data evoked by right ring finger stimulus.

Figure 7 shows two reconstructions of the data formed using a single time sample (for each sensor) $36 \mathrm{~ms}$ after the stimulus. $7(\mathrm{c}, \mathrm{d})$ depict a reconstruction with a smaller weighting $(\lambda)$ on the image prior term. The active region of the brain for this experiment should be the left somatosensory cortex, located in the region above the left ear canal. Figure $7(\mathrm{a}, \mathrm{b})$, shows a strong preference of our model to select this region. On the other hand, less dependence on the irregular lattice GMRF image prior creates an image which is not as definitive, as shown in Figure $7(\mathrm{c}, \mathrm{d})$. Both solutions are equally consistent with the observed data, $\mathbf{b}$, which illustrates the need for a strong regularizing term in the reconstruction.

\section{CONCLUSIONS}

This paper has demonstrated the effectiveness of a vector form Gauss-Markov random field model for Maximum $a$ posteriori reconstruction of brain current dipole distributions from external magnetic field measurements. A technique was presented for achieving (approximately) an arbitrary desired covariance structure for a non-uniformly sampled Markov random field while keeping the neighborhood structure small. The experiments with real MEG data are encouraging. Solutions have reduced random noise, reduced canceling dipole content (i.e. dipole configuration components that are invisible at the sensors), and better resolution of isolated dipoles than an unregularized image reconstruction.

\section{REFERENCES}

[1] M. Singh, D. Doria, V.W. Henderson, G.C. Huth, and J. Beatty, "Reconstruction of images from neuromagnetic fields", IEEE Transactions on Nuclear Science, vol. NS-31, pp. 585-589, 1984.

[2] J. W. Phillips, R. M. Leahy, and J. C. Mosher, "MEGbased imaging of focal neuronal current sources", IEEE Transactions on Medical Imaging, vol. 16, no. 3, pp. 338-48, June 1997.
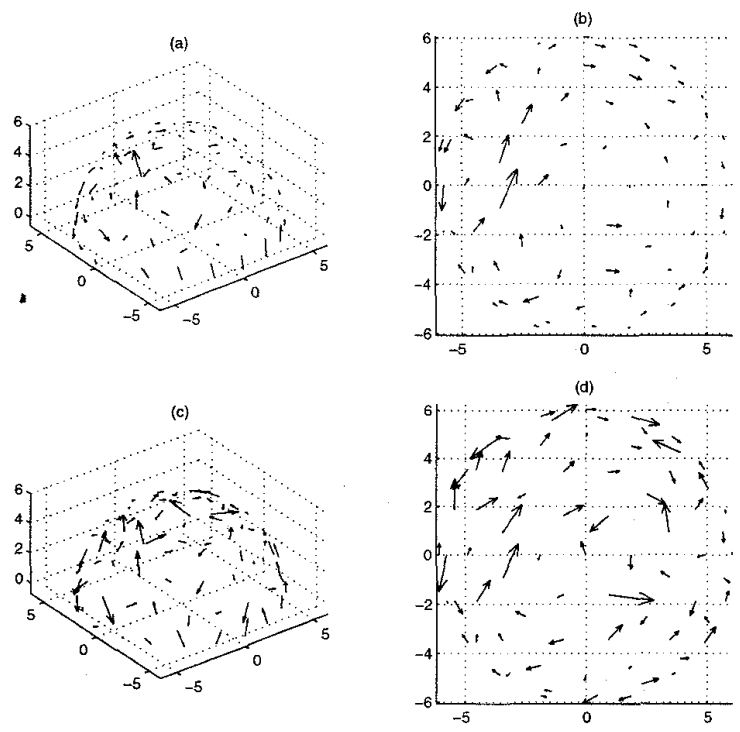

Figure 7. GMRF reconstructions of clinical MEG data. a) Side and, b) Top views. c) and d) Reconstruction with a less emphasis on the GMRF prior (i.e. $\lambda$ smaller than in $(a, b))$. The top of (b) and (d) corresponds to the forward section of the brain.

[3] S. Geman, K.M. Manbeck, and D.E. McClure, "A Comprehensive statistical model for single-photon emission tomography," in Markov Random Fields: Theory and Applications, R. Chellappa and A. Jain Eds., Academic Press, San Diego, 1993.

[4] J. W. Woods, "Two-dimensional discrete (markovian) fields", IEEE Transactions on Information Theory, pp. 232-240, Mar. 1972.

[5] Wai Ho Pun, Parametric Model-Adaptive Image Restoration, $\mathrm{PhD}$ thesis, Brigham Young University, 1995.

[6] C. Bouman and K. Sauer, "A generalized Gaussian image model for edge-preserving MAP estimation", IEEE Trans. on Image Processing, vol. 2, no. 3, pp. 296-310, July 1993.

[7] Alan H. Gardiner, "Map reconstruction of magnetoencepohalograms", Master's thesis, Brigham Young University, 1997.

[8] C. C. Chen, Markov random field models in image analysis, PhD thesis, Michigan State University, East Lansing, 1988. 